\title{
Searching for the 27-day solar rotational cycle in lightning events recorded in old diaries in Kyoto from the 17th to 18th century
}

\author{
Hiroko Miyahara ${ }^{1}$, Yasuyuki Aono ${ }^{2}$, and Ryuho Kataoka ${ }^{3,4}$ \\ ${ }^{1}$ Humanities and Sciences/Museum Carriers, Musashino Art University, 1-736 Ogawa-cho, Kodaira, \\ Tokyo 187-8505, Japan \\ ${ }^{2}$ Graduate School of Life and Environmental Sciences, Osaka Prefecture University, 1-1 Gakuen-cho, \\ Naka-ku, Sakai, Osaka 599-8531, Japan \\ ${ }^{3}$ National Institute of Polar Research, 10-3 Midori-cho, Tachikawa, Tokyo 190-8518, Japan \\ ${ }^{4}$ Department of Polar Science, School of Multidisciplinary Sciences, SOKENDAI, 10-3 Midori-cho, \\ Tachikawa, Tokyo 190-8518, Japan
}

Correspondence to: Hiroko Miyahara (miyahara@musabi.ac.jp)

Received: 28 August 2017 - Revised: 27 September 2017 - Accepted: 27 September 2017 - Published: 3 November 2017

\begin{abstract}
A solar rotational period of approximately 27 days has been detected in cloud and lightning activities, although the mechanism of the sun-climate connection remains unclear. In previous studies, lightning activity in Japan showed a significant signal of the solar rotational period, especially around the maxima of the decadal solar cycles. Here we analyze the time series of lightning activity in the AD 16681767 period, extracted from old diaries in Kyoto, Japan, and search for the signal of solar rotational cycles. The 27-day cycles were detected in the lightning data and occurred only around the maxima of the decadal sunspot cycles. The signal disappeared during AD 1668-1715, which corresponds to the latter half of the Maunder Minimum when both radiative and magnetic disturbances were thought to have been weak. These findings provide insight into the connection between solar activity and the Earth's climate.
\end{abstract}

Keywords. Meteorology and atmospheric dynamics (lightning)

\section{Introduction}

Correlations between solar cyclic activity and climate variations have been detected over a wide range of timescales, from monthly (Muraki et al., 2004; Sato et al., 2005; Takahashi et al., 2010; Hong et al., 2011; Scott et al., 2014), to decadal and multidecadal (Camp and Tung, 2007; Miyahara et al., 2008; Yamaguchi et al., 2010), to millennial (Bond et al., 2001; Obrocta et al., 2012). However, the detailed mechanisms of these connections are currently uncertain. Among the common cycles, the 27-day variability is of great importance because it offers the opportunity to understand how the sun influences weather and climate.

The apparent solar radiations detected on Earth, which are associated with solar rotations, alter over a 27 -day period (Coddington et al., 2016). The radiative outputs from the solar surface are controlled by the appearance and disappearance of sunspots and faculae, whereas the flux of open magnetic field is influenced by the appearance of sunspots and coronal holes (Lockwood, 2013). The magnetic outputs influence the flux of galactic cosmic rays incident on the Earth. Cosmic rays constitute a possible medium that connects the Sun and climate (Gray et al., 2010). As the magnetic flux conveyed to the solar surface increases and decreases over a decadal scale, the intensity of the 27-day component in the solar-related parameters varies over time.

Previously, we analyzed the lightning data over the AD 1989-2015 period and found a significant 27-day periodicity in the summertime wide-area lightning activity in Japan, especially around the maxima of the 11-year solar cycles (Miyahara et al., 2017a). The signal was strongest at a spatial coverage of at least a few hundred thousand square kilometers (covering more than 15 prefectures). A 27-day signal also appeared in the minima of the solar cycles, but the strength was approximately half that of the solar maxima. 
In modern times, the abovementioned 27-day signal shows a northeastward migration pattern, suggesting that the impact of solar activity propagates to Japan from lower latitudes. Consistent with this finding, the 27-day period in cloud activities is most significant in tropical regions (Takahashi et al., 2010; Hong et al., 2011). Therefore, the equatorial region may enable us to trace the solar influence on weather and climate.

In addition to examining the spatial extent of the 27-day periodicity in cloud and lightning activities, we must carefully test the temporal variations in the solar 27-day period in the meteorological phenomena associated with the centennial-scale high and low solar activities.

During the last decades, solar activity has rapidly trended downward. This trend may be a precursor of the coming Grand Minimum (e.g., Lockwood, 2013). The behavior of cyclic lightning activity over grand minima (such as the Maunder Minimum) might establish whether or not the 27-day periodicity becomes less significant during longterm low solar activity. For example, coronal mass ejections (CMEs), the major source of modulations in galactic cosmic rays on daily to monthly timescales, are expected to rarely occur during grand minima. High-speed solar wind streams from coronal holes may maintain cyclical behavior under such conditions, as was the case during the recent solar minimum, and it may affect the lightning activities via modulating energetic protons (Scott et al., 2014). However, Owens et al. (2017) estimate that the band of slow solar wind expanded to cover a sufficiently wide range of solar latitudes during the Maunder Minimum that the Earth continued to remain in the slow solar wind; the influence of any corotating fast streams had been minimized. The monthly-scale radiation changes should also be reduced due to the disappearance of sunspots. If radiation or galactic cosmic rays can generate the 27-day cyclic behavior of lightning, then such solar 27-day signals should be absent during the Grand Minimum.

This paper traces the possible behavioral transition of the 27-day periodicity in the lightning activities in Kyoto from the mid-17th to the mid-18th century, which includes the Maunder Minimum. The data were extracted from old diaries written in Kyoto. Section 2 describes the dataset and analysis method, Sect. 3 presents the results and discussion, and the concluding remarks are summarized in Sect. 4.

\section{Analysis methods}

Thunder and lightning events are frequently recorded in old diaries. During the Edo era (1603-1868), the weather information was often regularly recorded in priestly diaries. By examining these diaries, we can further explore the characteristics of the 27-day periodicity in lightning occurrences during long-term high and low solar activity. Recently we extracted a 100-year continuous record of summertime lightning events from several famous old diaries written in Kyoto
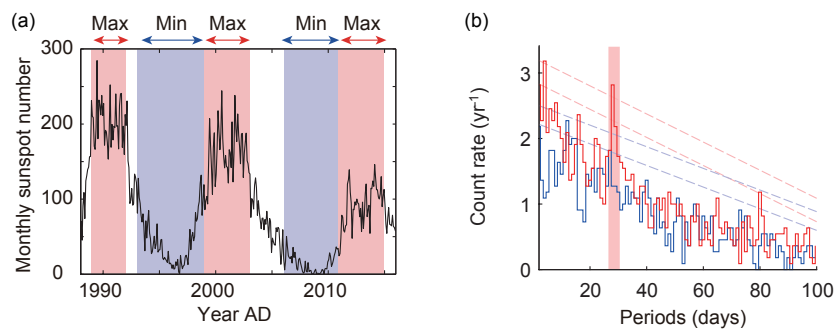

Figure 1. (a) Time series of monthly sunspot numbers during AD 1989-2015. The red and blue shading defines the periods of solar maxima and minima, respectively, used in the analyses. (b) Periodicity of lightning events from May to August in Kyoto during the solar maxima (red line) and minima (blue line). The red and blue dashed lines denote the 2 and $3 \mathrm{SD}$ in the solar maxima and minima, respectively. The red shaded bars indicate the 27-30-day period.

from the 17 th to the 18th century, which motivated us to examine the periodicity of lightning days. The record covers the latter half of the Maunder Minimum and the era of relatively high solar activity after recovery from the Maunder Minimum.

Following the method of Miyahara et al. (2017a), we conducted a histogram analysis of the lightning record. As discussed in their paper, the phases of the 27-day periodicities in solar-related parameters (such as solar radiation and galactic cosmic rays) change in a random manner and are associated with newly emerged sunspots and coronal holes. Therefore, we analyze the frequency distribution of time intervals by counting the number of days between every combination of two lightning days. This analysis reveals the typical waiting time of lightning days.

To examine the long-term characteristics of the 27-day signal in lightning activities, we consulted five old diaries stored in Kyoto. According to the previous study, the 27-day periodicity was more significant in wide-area lightning activity than local lightning, but some of the prefectures (including Kyoto) retained a relatively significant signal. The frequency spectrum over the AD 1989-2015 period in Kyoto, obtained by using the Miyahara et al. (2017a) method, revealed a 27day periodicity in the summertime lightning activity (Fig. 1). Similarly to the results of Miyahara et al. (2017a), the signal was more significant at the maxima of the solar decadal cycles (3.5 SD) than at the minima (2.1 SD).

In diaries of the typical Japanese style, the daily weather conditions are described before the daily occurrences are recorded. To analyze the lightning dates in the 17th to 18th centuries, we investigated the weather records in the diaries kept by Tsuneko Konoe (parts of AD 1668-1694), the Myoho-in Temple (parts of AD 1695-1767), the Nijo family (parts of AD 1728-1730), and the Chion-in Temple (parts of AD 1731-1767). The reliability of the daily weather descriptions from AD 1679-1697 was confirmed by consulting the diary of Kaneteru Ichijo. When the records of two diaries 


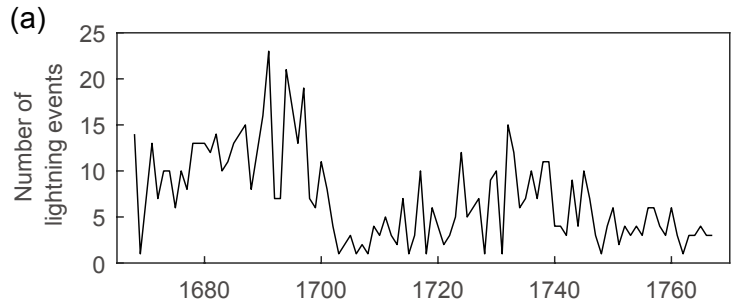

(b)

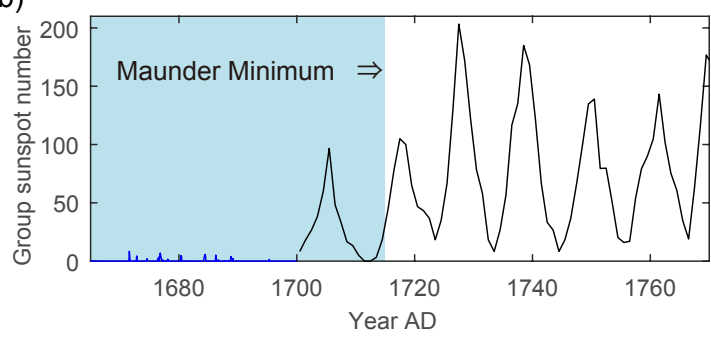

Figure 2. (a) Number of lightning events from May to August during AD 1668-1767 excavated from five old diaries in Kyoto, Japan. Note that the long-term differences in the numbers of lightning events in the 17th and 18th centuries may not exactly reflect the actual lightning activity level, as mentioned in the text. (b) Sunspot numbers over the same period (Clette and Lefevre, 2016, black line; Hoyt and Schatten, 1998, blue line). Blue shading indicates the period of the Maunder Minimum (Eddy, 1976).

overlapped, we assumed that the data were mutually supplementary and acquired a composite dataset of the overlapped lightning dates.

The abovementioned diary keepers appeared to differ in their "sensitivities" to natural phenomena (in this case, lightning). Some of the diary keepers noticed weak lightening events in both daytime and nighttime and recorded these trivial phenomena in their diaries. We noticed that the temple diary of Myoho-in and the private diaries of Tsuneko Konoe and Kaneteru Ichijo presented more detailed weather conditions and their temporal transitions than the other diaries. The family and temple diaries tended to be kept over longer periods than private diaries because the former were maintained over successive generations. To obtain longer time series of lightning dates in Kyoto, we have no choice but to acquire composite data from several diary keepers despite their various sensitivities to natural phenomena. Owing to the composite data, the change in the absolute number of lightning dates cannot be regarded as a long time series with homogeneous features.

Figure 2 shows the number of summertime lightning records during the AD 1668-1767 period extracted from the abovementioned old diaries. For comparison, the group sunspot numbers (Clette and Lefevre, 2016; Hoyt and Schatten, 1998) over the same period are shown in Fig. 2b. The weakening of the long-term solar activity during AD 16451715 is called the Maunder Minimum (Eddy, 1976). The
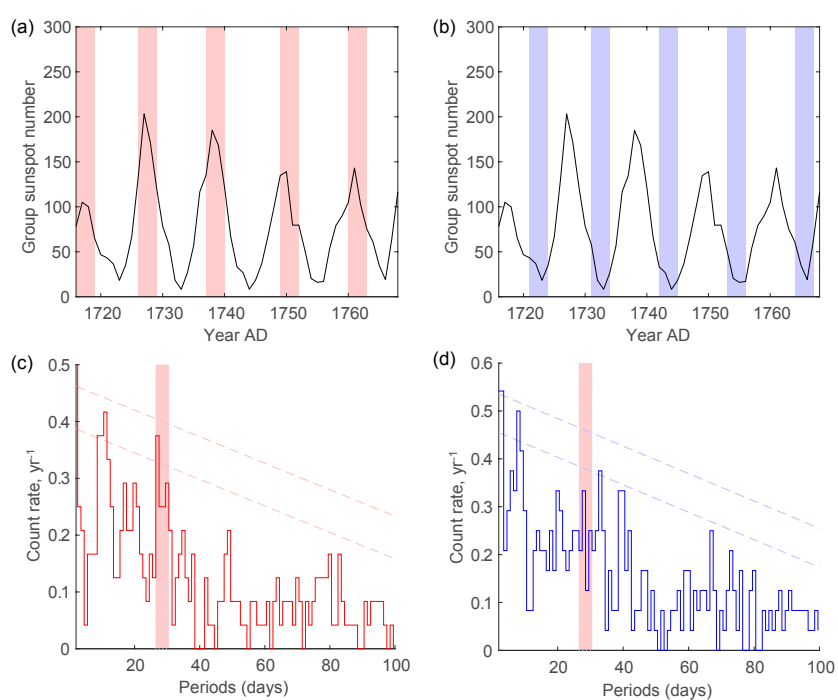

Figure 3. (a, b) Time series of group sunspot numbers for AD 1716-1767. The red and blue shading defines the periods of solar maxima and minima, respectively, used in the analyses. (c) Periodicity of lightning events during solar maxima. The red dashed lines denote 2 and 3 SD. The red shaded bar indicates the 27-30-day period. (d) Same as in panel (c) but for solar minima.

sunspot activity began to recover around AD 1700 and ended around $\mathrm{AD} 1715$.

\section{Results and discussion}

The characteristics of the 27-day cycles depend on the phase of the solar decadal cycles. Therefore, we divided the whole period into periods of high and low solar activities based on the group sunspot numbers. In the AD 1716-1767 period, we identified the maxima and minima of sunspot numbers from the yearly data compiled by Clette and Lefevre (2016). In our definition, the maxima lasted from 1 year before the peak to 2 years after the peak, whereas the minima lasted from 2 years before the peak to 1 year after the peak (Fig. 3a, b).

The maxima and minima of the solar activity cycles in the Maunder Minimum were derived from the carbon-14 content in tree rings. Carbon-14 data from tree rings, which can be precisely dated by dendrochronology, provide accurate timing for solar activity changes. The solar cycles apparently continued throughout the Maunder Minimum with an approximate period of 14 years (Miyahara et al., 2004). The periods of the solar maxima and minima were determined from the band-pass-filtered carbon-14 data in tree rings compiled by Stuiver et al. (1998). To accommodate the delay in the carbon cycle relative to the solar activity (Siegenthaler et al., 1980), the filtered curve was shifted by 2 years. As detailed in Kataoka et al. (2017), this method accurately detects the solar maxima and minima in solar cycles 1-18 with an uncertainty of \pm 1.7 years. When the solar cycles lengthen, the solar cycle 
(a)

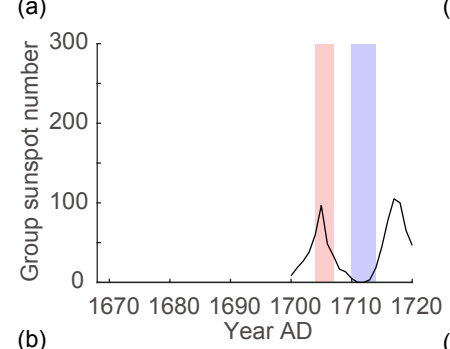

(b)

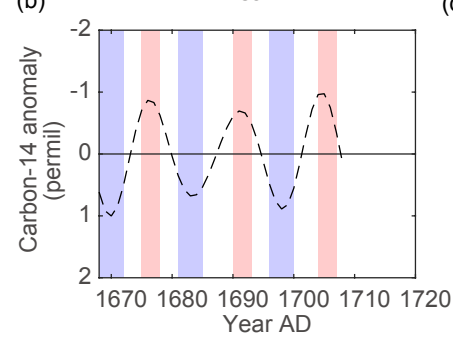

(d)
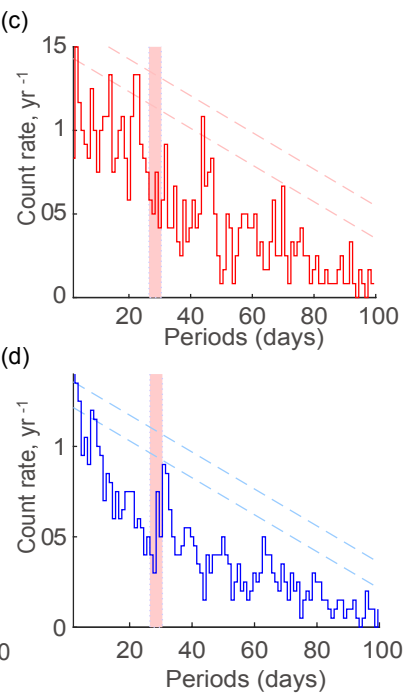

Figure 4. (a) Group sunspot numbers around the latter half of the Maunder Minimum. (b) Solar cycles reconstructed from the carbon14 content in tree rings. The red and blue shading denotes the periods of solar maxima and minima, respectively, used in the analyses. (c) Periodicity of lightning events during the solar maxima shown in panel (b). The red dashed lines denote 2 and 3 SD during the solar maxima, and the red shaded bar indicates the 27-30-day period. (d) Same as in panel (c) but for solar minima.

signal is delayed by slightly longer than 2 years. Nevertheless, the solar cycle maxima reconstructed by using the above method successfully reproduce the solar cycle maxima at the end of the Maunder Minimum with no dating error (Fig. 4b). We changed the definition of the periods of solar minima in the Maunder Minimum as lasting from 2 years before the peak to 2 years after the peak; this is 1 year longer than the case for AD 1716-1767 owing to the lengthened solar cycles.

We first examine the lightning activities after the Maunder Minimum. For this purpose, we selected the 52 years from $\mathrm{AD} 1716$ to 1767 when the solar cyclic activity recovered to a relatively active state (see Fig. 2b). The 27-day solar rotational period in lightning activity displayed a significant (2.6 SD) signal from May to August during the solar maxima (Fig. 3c) but not during the solar minima (1.4 SD; see Fig. 3d). This result is consistent with modern observations, in which a solar 27-day periodicity of lightning events appears during the maxima of solar decadal cycles.

Interestingly, the significance level of the 27-day period was reduced when the data were analyzed for the May to September period (see Fig. S1 in the Supplement). At both solar maximum and solar minimum, the peaks at around 2730 days were insignificant (1.3 and 1.6 SD, respectively). In contrast, both peaks in this period are significant in the modern era, regardless of whether the September data are included or excluded.

The discrepancy between the modern era and the 18th century may be related to the climatological conditions in the

earlier century. This period corresponds to the late stage of the Little Ice Age when Japan's climate might have been more strongly influenced by the cold Siberian air mass. In the modern era, the Siberian air mass advances over Japan around October to March. Even in early autumn (September), the advance of this cold air mass causes frequent local updrafts in Kyoto and may dilute the 27-day signal propagated from lower latitudes. As discussed in Miyahara et al. (2017a), the 27-day signal is apparent only in the component propagated from lower latitudes and is absent during the season of dominant activity of the Siberian air mass. The local draft increases the noise level of random lightning events, possibly lowering the significance level of the solar rotational cycle.

Second, we examine the lightning activities during the Maunder Minimum. For this purpose, we selected the time interval from AD 1668 to 1715 . The solar cycles were determined from the carbon-14 record as mentioned above. During the solar maxima, the periodicity of lightning activity peaked at around 22-23 days and at 44 days; however, the signal of 27-30-day periodicity is not significant. During the solar minima, the spectrum peaks at around 31-32 days (1.9 SD), but the signal of 27-30-day periodicity is insignificant. Although the decadal-scale cycles of solar activity continued during the Maunder Minimum, the signal of the solar 27-day modulation in lightning activity was lost. As mentioned above, the uncertainty in the selection of the solar maxima and minima during the Maunder Minimum is $\pm 1-$ 2 years. However, this uncertainty does not affect the significance level of the peak at 27-30 days or the main results.

The disappearance of the 27-day modulation during the Maunder Minimum might be explained by the lack of CMEs or cyclic disturbances in the radiative output during that period. As CMEs are among the main modulators of galactic cosmic rays incident to the Earth, their absence diminishes the Forbush decrease and hence the monthly-scale variations of cosmic rays.

In future work, the appearance and disappearance of the 27-day solar rotational period in lightning activity should be examined at centennial timescales. For this purpose, we can consult old diaries over wide regions of Japan. Suitable target periods are the Grand Minimum prior to the Maunder Minimum and the Medieval Maximum. It is also important to test the spatial extent of the 27-day signal. This can be done, for instance, by accessing the records in different prefectures of Japan and in nearby countries and by following the propagation of the signal within the area (which has migrated northeastward over Japan during the modern era). Testing the propagation of the signal and identifying its origin (possibly at lower latitudes) would help to elucidate the effect of solar activity on the Earth's climate. 


\section{Conclusions}

We retrieved a summertime lightning record covering the 100-year period from AD 1668 to 1767. The data were extracted from five old diaries in Kyoto, Japan. The impact of the 27-day solar rotational cycle on lightning periodicity was evaluated. Similarly to the modern era, the 27-day solar periodicity was significantly replicated in the lightning record of the 18th century after recovery from the Maunder Minimum. Also similarly to the modern era, the signal was significant only during the maxima of the solar decadal cycles. The signal of the 27-day periodicity completely disappeared during the Maunder Minimum when both radiative and magnetic disturbances may have been absent.

Data availability. The lightning data used in this study are available online at https://doi.org/10.6084/m9.figshare.5544841.v1 (Miyahara et al., 2017b).

\section{The Supplement related to this article is available online at https://doi.org/10.5194/angeo-35-1195-2017- supplement.}

Competing interests. The authors declare that they have no conflict of interest.

Acknowledgements. This work was supported by JSPS KAKENHI grant numbers $15 \mathrm{H} 05816$ and 25287051 . This work was also supported by the Center for the Promotion of Integrated Sciences of SOKENDAI and the Project to Build an International Collaborative Research for Pre-modern Japanese Texts. We thank Chika Higuchi, Chie Ogawa, and Kana Sakaguchi for their help in organizing the lightning dataset.

The topical editor, Marc Salzmann, thanks Chris Scott for help in evaluating this paper.

\section{References}

Bond, G., Kromer, B., Beer, J., Muscheler, R., Evans, M. N., Showers, W., Hoffmann, S., Lotti-Bond, R., Hajdas, I., and Bonani, G.: Persistent solar influence on North Atlantic climate during the Holocene, Science, 294, 2130-2136, 2001.

Camp, C. D. and Tung, K. K.: Surface warming by the solar cycle as revealed by the composite mean difference projection, Geophys. Res. Lett., 34, L14703, https://doi.org/10.1029/2007GL030207, 2007.

Clette, F. and Lefevre, L.: The new sunspot number: assembling all corrections, Sol. Phys., 291, 2629-2651, https://doi.org/10.1007/s11207-016-1014-y, 2016.

Coddington, O., Lean, J. L., Pilewskie, P., Snow, M., and Lindholm, D.: A solar irradiance climate data record, B. Am. Meteorol. Soc., 97, 1265-1282, https://doi.org/10.1175/BAMS-D-14$00265.1,2016$.
Eddy, J. A.: The Maunder Minimum, Science, 192, 1189-1202, 1976.

Gray, L. J., Beer, J., Geller M., Haigh, J. D., Lockwood M., Matthes, K., Cubasch, U., Fleitmann, D., Harrison, G., Hood, L., Luterbacher, J., Meehl, G. A., Shindell, D., van Geel, B., and White, W.: Solar influence on climate, Rev. Geophys., 48, RG4001, https://doi.org/10.1029/2009RG000282, 2010.

Hong, P. K., Miyahara, H., Yokoyama, Y., Takahashi, Y., and Sato, M.: Implications for the low latitude cloud formations from solar activity and the quasi-biennial oscillation, J. Atmos. Sol.-Terr. Phy., 73, 587-591, 2011.

Hoyt, D. V. and Schatten, K. H.: Group sunspot numbers: A new solar activity reconstruction, Sol. Phys., 181, 491-512, 1998.

Kataoka, R., Isobe, H., Hayakawa, H., Tamazawa, H., Kawamura, A. D., Miyahara, H., Iwahashi, K., Yamamoto, K., Takei, M., Terashima, T., Suzuki, H., Fujiwara, Y., and Nakamura, T.: Historical space weather monitoring of prolonged aurora activities in Japan and in China, Space Weather, 15, 392-402, https://doi.org/10.1002/2016SW001493, 2017.

Lockwood, M.: Reconstruction and prediction of variations in the open solar magnetic flux and interplanetary conditions, Living Rev. Sol. Phys., 10, 1-88, https://doi.org/10.12942/lrsp-2013-4, 2013.

Miyahara, H., Masuda, K., Muraki, Y., Furuzawa, H., Menjo, H., and Nakamura, T.: Cyclicity of solar activity during the Maunder Minimum deduced from radiocarbon content, Sol. Phys., 224, 317-322, 2004.

Miyahara, H., Yokoyama, Y., and Masuda, K.: Possible link between multi-decadal climate cycles and periodic reversals of solar magnetic field polarity, Earth Planet. Sci. Lett., 272, 290-295, 2008.

Miyahara, H., Higuchi, C., Terasawa, T., Kataoka, R., Sato, M., and Takahashi, Y.: Solar 27-day rotational period detected in widearea lightning activity in Japan, Ann. Geophys., 35, 583-588, https://doi.org/10.5194/angeo-35-583-2017, 2017a.

Miyahara, H., Aono, Y., and Kataoka, R.: Date of thunder and lightning events in Kyoto, Japan, available at: https://doi.org/10.6084/ m9.figshare.5544841.v1, last access: 2 November 2017b.

Muraki, Y., Axford, W. I., Matsubara, Y., Masuda, K., Miyamoto, Y., Menjyou, H., Sakakibara, S., Sako, T., Takami, T., Yamada, T., Shibata, S., Munakata, Y., Munakata, K., Yasue, S., Sakai, T., Mitsui, K., Fujimoto, K., and Flückiger, E.: Effects of atmospheric electric fields on cosmic rays, Phys. Rev. D, 69, 123010, https://doi.org/10.1103/PhysRevD.69.123010, 2004.

Obrochta, S. P., Miyahara, H., Yokoyama, Y., and Crowley, T. J.: A re-examination of evidence for the North Atlantic "1500-year cycle” at Site 609, Quat. Sci. Rev., 55, 23-33, 2012.

Owens, M. J., Lockwood, M., and Riley, P.: Global solar wind variations over the last four centuries, Sci. Rep., 7, 41548, https://doi.org/10.1038/srep41548, 2017.

Sato, M. and Fukunishi, H.: New evidence for a link between lightning activity and tropical upper cloud coverage, Geophys. Res. Lett., 32, L12807, https://doi.org/10.1029/2005GL022865, 2005.

Scott, C. J., Harrison, R. G., Owens, M. J., Lockwood, M., and Barnard, L.: Evidence for solar wind modulation of lightning, Environ. Res. Lett., 9, 055004, https://doi.org/10.1088/17489326/9/5/055004, 2014. 
Siegenthaler, U., Heimann, M., and Oeschger, H.: C-14 variations caused by changes in the global carbon-cycle, Radiocarbon, 22, 177-191, 1980.

Stuiver, M., Reimer, P. J., and Braziunas, T. F.: High-precision radiocarbon age calibration for terrestrial and marine samples, Radiocarbon, 40, 1127-1151, 1998.

Takahashi, Y., Okazaki, Y., Sato, M., Miyahara, H., Sakanoi, K., Hong, P. K., and Hoshino, N.: 27-day variation in cloud amount in the Western Pacific warm pool region and relationship to the solar cycle, Atmos. Chem. Phys., 10, 1577-1584, https://doi.org/10.5194/acp-10-1577-2010, 2010.
Yamaguchi, Y. T., Yokoyama, Y., Miyahara, H., Sho, K., and Nakatsuka, T.: Synchronized northern hemisphere climate change and solar magnetic cycles during the maunder minimum, P. Natl. Acad. Sci. USA, 107, 20697-20702, 2010. 